\title{
Relación entre la comunicación interpersonal y el proceso de socialización en los alumnos, docentes, egresados y universidad, para mejorar el servicio educativo a distancia. Caso universidad privada de Argentina
}

\author{
Mg. Ing. Rafael Francisco Matías Visa \\ rafael.visa@gmail.com \\ Dr. José Antonio Hernández Gutiérrez \\ jantonio_hergut@hotmail.com \\ Universidad Internacional Iberoamericana \\ Salta - Argentina
}

\section{RESUMEN}

El auge de la Educación a distancia presenta beneficios que la diferencian de la educación presencial, como la flexibilidad de tiempos y espacios, apoyados en los avances tecnológicos respecto de la información y de la comunicación. Entonces: ¿Cuál es la relación entre la comunicación interpersonal y el proceso de socialización en los alumnos, docentes, egresados y Universidad, para mejorar el servicio educativo a distancia en la Universidad Privada de Argentina? El objetivo general es analizar la relación entre la comunicación interpersonal y el proceso de socialización entre las partes mencionadas, para mejorar el servicio educativo a distancia. Se opta por una metodología mixta (cuantitativa y cualitativa), siendo la técnica de recolección de datos el cuestionario. Los principales resultados de este estudio señalan que la comunicación interpersonal a distancia se caracteriza por una constante interrelación mediada por recursos tecnológicos. Se lleva adelante el proceso de socialización a través del cambio de rol entre docentes y alumnos. Existe una relación entre la comunicación interpersonal y el proceso de socialización, puesto que, desde la organización escolar, se incorporaron modelos, de comunicación, adecuados a los avances tecnológicos actuales. Las estrategias innovadoras se implementan a los fines de mejorar el servicio educativo a distancia.

Palabras Claves: comunicación interpersonal; proceso de socialización; servicio educativo a distancia. 


\title{
Relationship between interpersonal communication and the
} socialization process in students, teachers, graduates and University, to improve distance education service. Private University

\section{of Argentina case}

\begin{abstract}
The rise of distance education presents benefits that differentiate it from face-to-face education, such as the flexibility of times and spaces, supported by technological advances regarding information and communication. So: What is the relationship between interpersonal communication and the socialization process in students, teachers, graduates and the University, to improve the distance educational service at the Private University of Argentina? The general objective is to analyze the relationship between interpersonal communication and the socialization process between the mentioned parties, in order to improve the distance educational service. A mixed methodology (quantitative and qualitative) is chosen, the data collection technique being the questionnaire. The main results of this study indicate that distance interpersonal communication is characterized by a constant interrelation mediated by technological resources. The socialization process is carried out through the change of role between teachers and students. There is a relationship between interpersonal communication and the socialization process, since, from the school organization, communication models were incorporated, adapted to current technological advances. Innovative strategies are implemented in order to improve the distance educational service.
\end{abstract}

Keywords: interpersonal communication; socialization process; distance educational service.

Artículo recibido: 20. Julio. 2021 Aceptado para publicación: 18. Agosto. 2021

Correspondencia: rafael.visa@gmail.com Conflictos de Interés: Ninguna que declarar 


\section{INTRODUCCIÓN}

En la actualidad, ha surgido el auge de la educación a distancia, porque posee una serie de beneficios que la diferencia de la educación presencial. Entre estos se pueden mencionar la flexibilidad de los tiempos utilizados por los alumnos para estudiar, como así también otros espacios de estudio. Estos beneficios mencionados, se convierten en una oportunidad para quienes viven alejados de las zonas urbanas, o poseen escaso tiempo para dedicarle al estudio por su horario laboral, lo cual se presenta como un obstáculo al momento de concurrir a las clases presenciales.

Para ello, a los fines de satisfacer las expectativas de los alumnos, es necesario que, desde las Universidades, se brinden programas eficientes, eficaces y que, tengan en cuenta los avances tecnológicos.

Ello, es de vital importancia puesto que, estos avances respecto de la información y de la comunicación, incorporando estos avances en nuevos paradigmas sobre cómo aprender, a los fines de transformar el proceso de aprendizaje, el rol del alumno, haciéndolo partícipe de la propia construcción de su conocimiento, teniendo en cuenta a su vez, las relaciones interpersonales entre los actores de la comunidad educativa y su relación con el proceso de socialización entre dichos actores.

\section{A partir de lo expuesto, se formula el siguiente problema de investigación:}

- ¿Cuál es la relación entre la comunicación interpersonal y el proceso de socialización en los alumnos, docentes, egresados y Universidad, para mejorar el servicio educativo a distancia en la Universidad Privada de Argentina?

\section{A partir de este interrogante, se sistematiza el problema de investigación, a través de} las siguientes preguntas:

- ¿Cómo se caracteriza la comunicación interpersonal entre alumnos, docentes, egresados y la Universidad Privada de Argentina mediante la modalidad a distancia?

- ¿De qué manera se lleva adelante el proceso de socialización entre alumnos, docentes, egresados y la Universidad Privada de Argentina mediante la modalidad a distancia?

- ¿Cómo se relaciona en la Universidad Privada Argentina, la comunicación interpersonal y el desarrollo proceso de socialización entre alumnos, docentes, egresados? 
- ¿Qué estrategias innovadoras se implementan a los fines de mejorar el servicio educativo a distancia en la Universidad Privada de Argentina?

El propósito de esta investigación, es mejorar la relación entre la comunicación interpersonal y el proceso de socialización en los alumnos, docente, egresados y el resto del personal de la universidad a los fines también de elevar la calidad del servicio educativo a distancia.

\section{ESTRATEGIAS METODOLÓGICAS O MATERIALES Y MÉTODOS}

Para realizar esta investigación, se recurrió a una metodología mixta. Mediante la metodología cuantitativa, se examinarán los datos de manera numérica, una vez implementada la encuesta apelando al campo de la estadística, según lo señalan Yuni y Urbano (2014), y por medio de la metodología cualitativa, se describirán las cualidades del fenómeno objeto de estudio de esta investigación. Se realizó una investigación descriptiva, es decir tendiente a la descripción del objeto de estudio.

La población de estudio, está compuesta por docentes, alumnos y egresados de la Universidad Privada Nacional, de la carrera de Licenciatura en Gestión Educativa, correspondiendo a un total de 500 personas.

La muestra se halla conformada en total por 217 personas. La técnica para calcular el tamaño de la muestra es la siguiente:

Para calcular el tamaño de la muestra se utilizó la siguiente fórmula:

$$
\bar{x}-z \frac{\sigma}{\sqrt{n}} \sqrt{\frac{N-n}{N-1}} \leq \mu \leq \bar{x}+z z \frac{\sigma}{\sqrt{n}} \sqrt{\frac{N-n}{N-1}}
$$

\section{Donde:}

$\mathbf{n}=$ el tamaño de la muestra.

$\mathbf{N}=$ tamaño de la población.

$\boldsymbol{\sigma}=$ Desviación estándar de la población que, generalmente cuando no se tiene su valor, suele utilizarse un valor constante de 0,5 .

$\mathbf{Z}=$ Valor obtenido mediante niveles de confianza. Es un valor constante que, si no se tiene su valor, se lo toma en relación al 95\% de confianza equivale a 1,96 (como más usual) o en relación al $99 \%$ de confianza equivale 2,58 , valor que queda a criterio del investigador. 
$\mathbf{e}=$ Límite aceptable de error muestral que, generalmente cuando no se tiene su valor, suele utilizarse un valor que varía entre el $1 \%(0,01)$ y $9 \%(0,09)$, valor que queda a criterio del encuestador.

Ordenando se obtiene la fórmula para calcular el tamaño de la muestra:

$$
n=\frac{Z^{2} \sigma^{2} N}{e^{2}(N-1)+Z^{2} \sigma^{2}}
$$

Se tiene $N=500$, para el $95 \%$ de confianza $Z=1,96$, y como no se tiene los demás valores se tomará y e $=0,05$.

Reemplazando valores de la fórmula se tiene:

$$
n=\frac{1.96^{2} 0.5^{2} 500}{0.05^{2}(500-1)+1.96^{2} 0.5^{2}}
$$

De la muestra determinada (217), se considerarán en la misma a 17 docentes que componen la planta de la Universidad, seleccionados en forma aleatoria para mejorar la calidad de la misma, considerando diferentes facultades y carreras. Del grupo de alumnos, se considerarán a 150 distribuidos en las diferentes unidades académicas disponibles en las diferentes regiones del País. Esto lleva a considerar datos provenientes de diferentes zonas geográficas, lo que implica situaciones cambiantes.

Del grupo de egresados, se evaluarán a 50 de los mismos, de diferentes carreras y unidades académicas geográficas, a los efectos de considerar variables que son afectadas por las zonas geográficas (tasa de empleo, tasa de pasantías, etc.). La muestra es de tipo probabilístico, ya que fue seleccionada en forma aleatoria (Sampieri, 2014). Por lo tanto, toda la población tuvo la misma posibilidad de ser elegida. Siendo la muestra a la vez, de tipo estratificada puesto que se seleccionó una muestra para el estrato jerárquico representado por docentes, una muestra representada por alumnos y finalmente otra muestra representada por egresados. Así, se señala que la muestra es de tipo probabilística estratificada.

Las variables independientes son las siguientes fueron: Atención al alumno; Escaso conocimiento sobre el uso de recursos tecnológicos; Toma de decisiones; Estrategias de publicidad y de venta y Calidad de servicio. A su vez, las variables dependientes son las siguientes:Fidelización de los alumnos y egresados a la Universidad; Reclamo de los alumnos, docentes y egresados y Satisfacción de los alumnos, docentes y egresados. 
Las técnicas de recolección de datos utilizada, fueron la encuesta y la observación.Los instrumentos de recolección de datos fueron elaborados ad hoc para esta investigación, y la forma en que se llevó adelante el proceso de validación del mismo fue aplicando la medida de estabilidad, lo cual significa que esos mismos instrumentos, se aplicaron en sucesivas oportunidades a un igual número de personas, durante un determinado tiempo, a los fines de establecer su confiabilidad por medio de test- retest, $r$ de Pearson.

\section{RESULTADOS Y DISCUSIÓN}

Por medio de la resolución 331/8, Universidad Privada, tramitó el Modelo Educativo del Sistema de Educación a Distancia SEAD.El mismo implica el posicionamiento teórico de la Universidad, en lo referente a la modalidad a distancia; es decir que los fundamentos del mismo se sustentan en los principios de la educación permanente, en la democratización de las oportunidades de aprendizaje y en la utilización de distintos medios, estrategias, materiales y nuevas tecnologías, que enmarcados didácticamente, promueven un proceso educativo autónomo, como una alternativa diferente. Por tal motivo, el modelo, adopta los siguientes principios:

- Educación permanente para todos a través de la planificación de proyectos educativos abiertos, que satisfagan demandas de formación, capacitación, actualización y perfeccionamiento, exigidos por las dinámicas transformaciones del medio social y económico. Amplia difusión democrática de las oportunidades de aprendizaje, atendiendo de modo flexible las expectativas de una población con distintas dificultades de acceso al sistema de educación superior.

- Favoreciendo la permanencia y culminación de las distintas instancias educativas.

- Utilización de distintos medios, estrategias, materiales y nuevas tecnologías, que enmarcados didácticamente, promuevan un proceso educativo autónomo, como una alternativa diferente.

- Asimismo los propósitos básicos que sustentan la educación a distancia en nuestra universidad son los siguientes:

Ha sido pionera en el país en la incorporación de la modalidad a distancia. Inicialmente, este sistema nace con la tradicional organización de tutorías en diferentes sedes. En el año 1998 el desarrollo tecnológico aportó un empuje fundamental al sistema, por lo que se generó la transmisión de audio y video por Internet y un entorno de aprendizaje mediante e-learning (aprendizaje electrónico). 
A partir del año 2006, comenzó un proceso de reorganización de las sedes distantes del Sistema de Educación a Distancia (SEAD), a los efectos de fortalecer una estructura académico-administrativa para favorecer el desarrollo coherente e institucional de las actividades de formación a distancia y la conducción centralizada del sistema.Para lograr este objetivo y provocar el mejoramiento cualitativo y cuantitativo del servicio educativo a distancia, el proyecto de unificación del servicio comprende la integración y mejor aprovechamiento de los recursos.

En la actualidad una carrera universitaria no es suficiente para satisfacer las cambiantes necesidades del mercado laboral, se requiere de una actitud de constante aprendizaje, cambio e innovación para lograr el crecimiento profesional. Por eso la Universidad Privada, acerca junto a los mejores exponentes de cada especialidad, esta oferta educativa de calidad, donde se encuentra las carreras adaptadas a los tiempos y exigencias.Funciona actualmente como referente de la Educación a Distancia en diversas instituciones, ya que se adecúa a los cambios constantes, dispone de estrategias pensadas para el docente y el alumno, y posee los medios de aprendizaje que permiten enriquecer y completar el proceso educativo.

Las Carreras a distancia son las siguientes: Licenciatura en Administración de Empresas; Contador Público- Licenciatura en Comercialización- Abogacía- Licenciatura en Gestión Educativa- Licenciatura en Relaciones Internacionales- Licenciatura en Educación Física - Ciclo de Complementación Curricular- Licenciatura en Seguridad - Ciclo de Complementación Curricular- Licenciatura en Economía- Licenciatura en Recursos Humanos- Licenciatura en Relaciones Públicas e Institucionales- Analista Universitario en Marketing- Analista Universitario en Recursos Humanos- Analista Universitario en Relaciones Públicas- Especialización en Gestión de las Tecnologías de la InformaciónEspecialización en Industria y Transformación Digital- Tecnicatura Universitaria en Secretariado Ejecutivo- Tecnicatura Universitaria en Higiene y Seguridad en el TrabajoTecnicatura Univ. en Gestión de Bancos y Empresas Financieras- Tecnicatura Universitaria en Administración de Asociaciones Civiles y Fundacion- Tecnicatura Universitaria en Ceremonial y Protocolo- Corredor Inmobiliario y Martillero PúblicoTecnicatura Universitaria en Seguros- Tecnicatura Universitaria en Gestión de Calidad

\section{Las estrategias para elevar la calidad del servicio, son las siguientes:}


- Atender, en general, a una población estudiantil dispersa geográficamente que no dispone de las redes de las instituciones educativas.

- Coordinar y administrar mecanismos de comunicación múltiples que permitan enriquecer los recursos del aprendizaje.

- Favorecer la posibilidad de mejorar la calidad de la instrucción al asignar la elaboración de los materiales didácticos a los mejores especialistas.

- Establecer la posibilidad de personalizar el proceso de aprendizaje para garantizar una secuencia académica que responda al ritmo de estudio del alumno.

- Promover la formación de habilidades para el trabajo independiente y para un esfuerzo autorresponsable.

- Formalizar vías de comunicación bidireccionales y frecuentes relaciones de mediación, dinámicas e innovadoras.

- Facilitar la permanencia del estudiante en su medio natural y cultural, con lo cual se evitan éxodos que inciden en el desarrollo regional.

Uno de los factores de la calidad de la educación a distancia es la capacidad de innovación, de perfeccionamiento, de superación de los problemas actuales:

- Por su método,

- Por su flexibilidad para facilitar distintos tipos de aprendizaje y responder a las demandas más variadas,

- Por el uso intensivo de los medios de comunicación y las nuevas tecnología de la información,

- Porque exigen el compromiso formal con el autoaprendizaje, que es a la vez condición y objetivo de toda formación de calidad.

- Mientras que a continuación se presentan las estrategias de interacción:

- Clases satelitales como medios complementarios del proceso de aprendizaje. Allí la interacción entre el profesor y los alumnos se desarrolla en tiempo real y son muy útiles para la realización de integraciones temáticas, estudio de casos, evaluaciones informales, etc.

- Una situación de aprendizaje interactivo implica una cadena de intervenciones de los participantes donde la comunicación adquiere la característica de multidireccionalidad; allí intervienen no sólo variables cognitivas sino también afectivas, metacognitivas y sociales, superando de esta manera, el intercambio de 
mensajes puntuales que sigue un patrón de preguntas y respuestas. En este sentido el aprendizaje va más allá de la búsqueda, adquisición y recuperación de información, sino que implica la gestión del conocimiento.

- El apoyo tutorial permanente es muy importante en el seguimiento del alumno. Puede definirse como un espacio didáctico, una estructura de apoyo a los estudiantes a distancia, en el que se realizan múltiples actividades con el fin de crear situaciones que favorezcan el proceso de aprendizaje. En este espacio el alumno tiene la oportunidad de sentirse acompañado por sus docentes, en un proceso de interacción permanente. Desde la perspectiva de los estudiantes, consiste en orientar y reorientar los procesos de comprensión y de transferencia. Desde el punto de vista de la enseñanza, los tutores:planifican su intervención docente a través de un diseño de actividades y encuentros presenciales y/o virtuales, donde se establezcan los contenidos, recursos y tiempos; desarrollan los ejes centrales de la asignatura; diseñan actividades complementarias que favorecen el estudio y desde una perspectiva más amplia e integradora, atienden a las situaciones y los programas particulares de cada uno de los alumnos: favorecen procesos de autoevaluación; las actividades que promueven las diversas estrategias de interacción deberán ir evolucionando, ajustándose en un primer nivel, desde un menor conocimiento del estudiante y una mayor dependencia del docente, a un estado de mayor conocimiento de los estudiantes y por lo tanto de mayor autonomía de los mismos, a través de un proceso de delegación de responsabilidad y control.

- El docente-tutor cuenta con un espacio muy importante de interacción sincrónica: los encuentros satelitales, que tienen como objetivo, presentar temas de actualidad relacionados con las asignaturas, desarrollar situaciones prácticas, foros, estudios de casos, evaluaciones de proceso. Son optativas. Este proceso de seguimiento y motivación que realiza el profesor tutor tanto en forma presencial como a través de diferentes espacios de comunicación por Internet, permite tener en cuenta en el diseño instruccional de los programas, los diferentes estilos de aprendizaje de los estudiantes y la forma cómo se pueden atender las necesidades diversas requeridas por los mismos.

- El foro será el espacio de trabajo que privilegiará la interacción de los actores del proceso, como el lugar de la conversación y del intercambio entre los estudiantes y el 
profesor y los estudiantes entre sí. Se prevé para este diseño distintos tipos de foros donde todos participan con sus intervenciones, de acuerdo a las exigencias de cada profesor con reflexiones, interrogantes, etc. Como la forma posible que la comunicación adopta a través del campus virtual es de mensajes de texto, éstos serán elaborados por los profesores para ayudar a construir el conocimiento, para incentivar a la producción de respuestas y para gestionar los productos de los trabajos grupales.

\section{Los recursos materiales multimediales y tecnológicos específicos, se presentan de la} siguiente manera:

Los alumnos tienen acceso a la información a través de diferentes materiales multimediales: módulos, bases de datos, lecturas complementarias, videos, audio, videoclases etc., disponibles en soporte impreso y en Internet.El módulo es el recurso principal de mediatización y se define como un conjunto integrado de contenidos, fuentes de información, actividades, a través del cual el docente implementa su programa de estudios y sus criterios y modalidades de evaluación que permiten permitan al alumno, «apropiarse» de los saberes que emergen de la bibliografía básica, fuente fundamental del conocimiento.Los módulos se presenten al alumno como una «conversación didáctica», en los cuales los mensajes enviados y recibidos son organizados de una manera clara, a través de ejemplos, casos de la vida cotidiana, que inviten al alumno a resolver cuestiones específicas y a exponer sus ideas.Deberá no solo sistematizar la información seleccionada para esa asignatura, sino además construir la presencia social del docente, enriqueciendo la comunicación en sus aspectos no verbales y paralingüísticos, restringidos por la asincronía del medio.Es importante recordar que el módulo es un verdadero mediador, no exclusivo, dentro del proceso de enseñanza y aprendizaje; un mediador que no «cierra» sino que problematiza e invita a buscar otras fuentes y a participar genuinamente en la construcción del conocimiento. Es importante destacar que la intervención intencional del profesor tiene en cuenta la especificidad de las tutorías y el tipo de andamiaje a realizar durante el proceso de enseñanza, de acuerdo a las posibilidades de interacción que ofrece el sistema. Considerar estos aspectos, permitirá potenciar la riqueza del campus virtual y compensar sus debilidades, intervención a la que se identifica conceptualmente como ayuda pedagógica, que puede realizarse a través de múltiples metodologías didácticas y siguiendo la evolución de los estudiantes, para poder graduarla hacia el conocimiento autónomo del mismo. 
La Universidad Privada, respecto de los recursos tecnológicos, se organiza de la siguiente manera:

- El Sistema de Educación a Distancia (SEAD) dispone con recursos tecnológicos que permiten la facilitación de contenidos académicos y gestiones administrativas, tanto del alumno como del profesor y del sistema de control.

- Tanto los encuentros satelitales como las videoclases, se desarrollan y distribuyen a través de transmisión de datos vía terrestre, conocido como "video streaming". Este es un complemento tecnológico de la plataforma virtual.

- Desde los estudios satelitales de Buenos Aires y Salta, los profesores se comunican con los estudiantes a través de su imagen y palabra, con videos complementarios, con el uso de diapositivas y otras opciones.

- En cada aula remota, la señal es recepcionada vía Internet en vivo, con calidad televisiva, lo que permite ampliar la imagen para la recepción del alumnado.

- En cada aula se cuenta con una terminal que permite a los estudiantes efectuar preguntas durante el desarrollo de la clase a través del Chat.

- Es importante destacar que ofrece también, a través de su prestador tecnológico, un enlace satelital de alta calidad de imagen como opción, para aquellas localidades que no cuenten con buena señal de Internet por tierra.

- Además, dispone de una plataforma de archivos (Plataforma Hermes), que permite tener disponibles las video clases o encuentros satelitales, después de emitida la señal en vivo, favoreciendo el acceso del alumno a la clase en diferido, en caso que no haya podido presenciarla en el momento de la transmisión.

\section{Otros sistemas informáticos importantes son:}

- Sistema de Autogestión (SAG) a través del cual el alumno realiza todas las consultas y transacciones administrativas respecto a la carrera, tales como inscripciones a exámenes, control de notas, etc.

- Sistema de Gestión Académica Administrativa (SIGAA) es un servicio exclusivo para empleados administrativos, coordinadores y tutores que les permite acceder de forma inmediata a una serie de consultas y transacciones académicas y administrativas de su Facultad, Unidad de Gestión o Tutoría desde cualquier PC conectada a Internet, durante las 24 hrs. todo el año.

Las competencias del docente en la educación a distancia, deben ser las siguientes: 
- Ser un facilitador del aprendizaje, a partir de diseños de experiencias que le ofrezcan al estudiante una estructura para el inicio de la interacción.

- Poseer competencias pedagógicas, didácticas y tecnológicas.

- Plantear tareas de enseñanza-aprendizaje abiertas que consigan activar en los estudiantes procesos cognitivos como la organización y elaboración del conocimiento.

- Desarrollar recursos suficientes en diferentes formatos a fin que puedan responder a las demandas que se realicen.

- Proponer un proceso de evaluación de los aprendizajes que ponga de manifiesto el resultado de la construcción de conocimiento por parte del estudiante.

- Establecer una cantidad importante de interacciones con los estudiantes para recoger información sobre el proceso y la calidad de la construcción de conocimientos que se vayan produciendo.

- Capacitarse para las intervenciones desde el modelo que se describe.

- Poseer habilidad para el manejo tecnológico en el campus virtual.

- Intervenir para facilitar las construcciones cooperativas y colaborativas de los alumnos.

Los resultados de las encuestas, en base a las variables independientes son las siguientes. El 66\% de los alumnos encuestados, señalan que respecto al servicio al alumno, el factor más importante es la interacción y en menor medida la confianza y el seguimiento. Respecto a la satisfacción del alumno, se sostiene que prevalece un buen servicio sobre el producto de calidad. En relación a la calidad del servicio, los alumnos que cursan distintas carreras en diferentes regiones del país, señalan que por sobre todo es importante la eficacia para que el servicio brindado por la universidad sea de calidad (57\%). En menor porcentaje señalan la rapidez y la flexibilidad. Respecto de los conocimientos tácitos, los alumnos cursantes, dan por sentado o por sabido que es de tácito conocimiento el intercambio personal (43\%) y la consultoría (41\%). El 64\% de los alumnos cursantes y encuestados, señalan que las publicaciones son importantes para determinar el conocimiento explícito al igual que los Congresos (61\%). Respecto de las decisiones acertadas, el $81 \%$ de los alumnos cursantes considera que es importante la comunicación y mínimamente el control (19\%). Al igual que en las decisiones desacertadas, los alumnos consideran que la más importante es la falta de comunicación (77\%). El 65\% piensa que 
la mejor estrategia de publicidad que pueda realizar la Universidad, y que puede llegar a una mayor audiencia, es la televisión (65\%) y mínimamente la prensa escrita (17\%) y la radio (10\%). El 97\% considera que la mejor estrategia de venta es Internet (97\%). El 87\% sostiene que la calidad del servicio se relaciona específicamente con el profesionalismo de los docentes. El 68\% de los alumnos encuestados y cursantes, señala respecto a la calidad del servicio, que la misma se encuentra relacionada a la empatía y directamente con la comunicación (68\%).

En relación a las variables dependientes, los resultados fueron los siguientes. Respecto de la fidelización de los alumnos y egresados a la universidad, los alumnos encuestados señalan que la retención del alumno, se da principalmente por medio de la calidad del servicio y en menor medida por la solución de sus reclamos. La fidelización de los alumnos y egresados a la universidad, en relación a la recuperación del alumno, está dada por la comunicación (43\%) y el aumento de beneficios (41\%) principalmente. Las quejas, en relación a los reclamos, se dan principalmente por parte de los alumnos (91\%). La percepción de los alumnos sobre la satisfacción es considerada muy buena (72\%) y sobresaliente en menor medida (23\%). El 75\% de los alumnos, se siente satisfecho con la calidad del servicio, mientras que un $25 \%$ se siente complacido. En relación a las características de la comunicación interpersonal entre alumnos, docentes, egresados y la Universidad Privada de Argentina mediante la modalidad a distancia, el 99\% de ellos, señala que se promueven las tecnologías de información y comunicación (TIC), es eficaz y eficiente en el entorno digital, que los estilos de comunicación de los docentes, condicionan, el tipo de interacción en el entorno virtual, y facilitan un buen clima de comunicación, que la comunicación y la interacción desarrollan una comunicación interpersonal, tendiente a facilitar el aprendizaje y la cohesión del grupo, mediante objetivos comunes y redes de aprendizaje y que el modelo de comunicación es la horizontal y dialógica.El 99\% de los alumnos egresados, señala que las características del proceso de socialización entre alumnos, docentes, egresados y la Universidad Privada de Argentina mediante la modalidad a distancia, son que el mismo es constructor de afinidades, permite la continuidad de vínculos y la formación continua. La relación existente en la Universidad Privada Argentina, entre la comunicación interpersonal y el desarrollo proceso de socialización entre alumnos, docentes, egresados, es muy positiva, caracterizándose por la comunicación interpersonal positiva y las óptimas posibilidades 
integradoras de la socialización. Las principales estrategias innovadoras que se implementan a los fines de mejorar el servicio educativo a distancia en la Universidad Privada de Argentina, son la construcción de su propia identidad, red de relaciones que se asientan en la virtualidad mayor uso de tecnologías de la información y comunicaciones y la capacitación de profesores (100\%) y en menor medida, un $99 \%$ de los egresados responde que otras estrategias son el acompañamiento del alumno en el proceso de cambio que implica ser un estudiante a distancia y la prestación de un buen servicio.

\subsection{DISCUSIÓN}

En la actualidad una carrera universitaria no es suficiente para satisfacer las cambiantes necesidades del mercado laboral, se requiere de una actitud de constante aprendizaje, cambio e innovación para lograr el crecimiento profesional. Por eso la Universidad Privada, acerca junto a los mejores exponentes de cada especialidad, esta oferta educativa de calidad, donde se encuentra las carreras adaptadas a los tiempos y exigencias.

En la educación a distancia, son de gran importancia, la mediación tecnológica, a través del uso multimedia, dando lugar así, a un aprendizaje autodirigido y autorregulado. Este modelo, depende de la ubicación de las conexiones en red entre las personas, los artefactos digitales y el contenido, lo que habría sido inconcebible como formas de educación a distancia donde la World Wide Web no estuviera disponible para mediar en el proceso. Así, como se ha visto en el caso de las generaciones anteriores de aprendizaje a distancia, la tecnología ha jugado un papel importante en la determinación de las pedagogías potenciales que pueden ser empleadas. Lo que diferencia la educación a distancia, de la educación tradicional, son la asincronicidad, aprendizaje autónomo, comunicación centrada en los medios tecnológicos, configurando redes de comunicación entre quienes participan del proceso de enseñanza y aprendizaje mediado por tutorías. De esta manera, y de acuerdo a su fundamento se apoya en el desarrollo de herramientas tecnológicas bajo los principios de libertad de uso, distribución, estudio y modificación, con la libertad para utilizar, difundir, aprender y adaptar cualquier tipo de material didáctico, siendo estos apuntes, libros de texto, presentaciones, programaciones o unidades didácticas, bibliografías, animaciones, simulaciones, vídeos, audios, entre otros, denominados Recursos Educativos Abiertos (Open EducationalResources).Por lo tanto, surge la necesidad de plantear distintas metodologías didácticas centradas en el alumno, 
una educación abierta y flexible que fomente la participación y autonomía del alumno en los procesos de aprendizaje mediados por TIC, puesto que la educación pone énfasis en el aprendizaje del alumno con la utilización de tecnologías.

Ante lo expuesto, se señala que este modelo, depende de la ubicación de las conexiones en red entre las personas, los artefactos digitales y el contenido, lo que habría sido inconcebible como formas de educación a distancia donde la World Wide Web no estuviera disponible para mediar en el proceso y se debe aclarar que los diferentes modelos de enseñanza y aprendizaje han evolucionado de manera paralela con la tecnología. La interacción y el diálogo son la base del proceso de enseñanza y aprendizaje, siendo así considerado líder de discusión, facilitador, facilitador de procesos y orientador del discurso, porque el docente debe tener la capacidad de dirigir el discurso y mantener motivado a los estudiantes en el desarrollo de los temas y la adquisición de las competencias, mediante intervenciones periódicas, realimentación y motivación, a través de mensajes o documentos escritos que se envían por medio de la plataforma tecnológica.

\section{CONCLUSIÓN O CONSIDERACIONES FINALES}

En la educación a distancia, la comunicación y la interacción desarrollan una comunicación interpersonal, tendiente a facilitar el aprendizaje y la cohesión del grupo, mediante objetivos comunes y redes de aprendizaje

Las características de la comunicación interpersonal entre alumnos, docentes, egresados y la Universidad Privada de Argentina mediante la modalidad a distancia, mediante la modalidad a distancia, promueve las tecnologías de información y comunicación (TIC), siendo la comunicación, eficaz y eficiente en el entorno digital y los estilos de comunicación de los docentes, condicionan, el tipo de interacción en el entorno virtual, y facilitan un buen clima de comunicación.

En dicho proceso, se plantean actividades que promueven el constructivismo y el aprendizaje colaborativo a fin de que los recién llegados, puedan aprender relacionando teoría y práctica, mientras que el equipo docente, interviene pedagógicamente a modo de guía y orientación para favorecer procesos de aprendizaje, en los cuales los estudiantes se van socializando en la vida académica, ganando autonomía en el uso de las tecnologías para formular y comunicar opiniones fundamentadas. Así, el proceso de socialización, se diseña y planifica articulando la teoría y la práctica. 
Para lograr la continuidad de los estudiantes, los docentes entablan una relación dialógica permanente (mediada a través de los recursos del Campus) correo electrónico, foros y espacios de comunicación en el aula; y que trabajen de modo articulado con el equipo de tutores académicos, sabiendo que la educación universitaria, es un proceso complejo, mediado por las tecnologías, integrado, que atiende a procesos y lógicas propias. Por este motivo, en la Universidad privada, se considera necesario pensar en el diseño de materiales de carácter abierto, que presenten flexibilidad, que permitan efectuar cambios periódicos (en el caso de que fuera necesario) considerando que cada grupo es diverso, que cada sujeto sabe o cree saber distintas cosas, que comprenden de maneras diferentes, que manejan tiempos diferentes y, a la vez, requieren de tiempos diferenciados para conocer, enmarcados en dimensión institucional universitaria.

Además, los docentes poseen capacidad de comprensión general, capacidad comunicativa y capacidad para evaluar pedagógicamente las situaciones y los procesos en los que se encuentran implicados. La mirada docente propicia la construcción de conocimiento legítimo sin dejar este hecho librado únicamente a los diseños instruccionales y el posicionamiento pedagógico parte de la idea de que la calidad de los materiales, tanto como del diseño, son importantes pero no necesariamente educativos por sí mismos. En ese sentido, la tarea de los docentes es ponerse a disposición para aclarar, explicar, re preguntar, citar ejemplos y pensar en mediaciones adecuadas para cada grupo y estudiante. El equipo docente deberá proponer y guiar para que se produzcan diferentes aprendizajes. Desde la perspectiva de los docentes en el proceso de socialización, también se producen tensiones entre el plano de lo ideal y el plano de la realidad. Por eso, se predisponen a entender quiénes están del otro lado, cuáles son sus necesidades, de qué manera aprenden y cuáles son sus metas, trabajando posicionados en el paradigma de la complejidad, reconociendo los múltiples saberes previos y orientándolos para que puedan ir asumiendo el rol de estudiante universitario.

La mirada institucional, en tanto, prioriza que la Universidad esté presente guiando a sus estudiantes y, en el caso de los docentes durante el proceso de socialización, continúe fomentando su formación permanente, fortalezca los espacios reflexivos para debatir ideas y proyectos, ajustar los materiales disponibles para que resulten significativos y autoevaluarse para recibir y enseñar adecuadamente a los recién llegados. De esta manera, 
el proceso de socialización, avanza y evoluciona hacia la construcción de afinidades que dependiendo de las habilidades sociales de los alumnos, dan origen a pequeños grupos que se reúnen como consecuencia de compartir intereses u objetivos comunes. En otras palabras, los compañeros dejan de ser todos iguales para ser unos más cercanos que otros, desde el inicio de dicho proceso. Ante lo expuesto, se puede señalar que esos vínculos conformados como consecuencia de la comunicación interpersonal, a la vez se relacionan con el proceso de socialización entre alumnos, docentes, egresados.

La comunicación interpersonal y el desarrollo proceso de socialización entre alumnos, docentes, egresados, es muy positiva, caracterizándose por la comunicación interpersonal positiva y las óptimas posibilidades integradoras de la socialización. Es por ello, que las tecnologías de información y comunicación (TIC) conllevan diferentes cambios propiciando el incremento de las relaciones sociales e intelectuales en el ámbito educativo y brindando oportunidades de nuevas posibilidades de aprendizaje e información. El impacto de las TIC, en el aspecto educativo, se utiliza como medio de expresión e instrumento para procesar información, como fuente de información, canal de comunicación interpersonal, así como recurso interactivo para el aprendizaje e un instrumento cognitivo. Ello indica que las TICs, se convierten en un instrumento indispensable en las instituciones educativas, puesto que ofrecen nuevos entornos para la enseñanza y el aprendizaje, libres de restricciones que imponen el tiempo y el espacio en la enseñanza presencial y aseguran una continua comunicación docente /participante. Así, los avances tecnológicos, impactaron en los procesos de enseñanza-aprendizaje, incorporando nuevas estrategias de aprendizaje y el empleo de herramientas como el Internet, la plataforma virtual, los espacios síncronos y asíncronos en la comunicación. De esta manera, surge un cambio social, dando lugar a la sociedad del conocimiento, al ampliar las capacidades físicas y mentales de sus integrantes. Estas transformaciones, son imprescindibles en la educación actual, contemplando un nuevo modelo de aprendizaje basado en cómo se concibe el proceso docente, el papel activo del alumno como constructor de su conocimiento, y de la interacción profesor-alumnos y alumno- alumno en el proceso educativo.

También, las principales estrategias innovadoras que se implementan a los fines de mejorar el servicio educativo a distancia en la Universidad Privada de Argentina, son la construcción de su propia identidad, red de relaciones que se asientan en la virtualidad 
mayor uso de tecnologías de la información y comunicaciones y la capacitación de profesores y en menor medida, el o acompañamiento del alumno en el proceso de cambio que implica ser un estudiante a distancia y la prestación de un buen servicio.Esta mejora del servicio educativo a través de la implementación de estrategias innovadoras, llevan a la satisfacción del alumno, lo cual se logra mediante una buena implementación de aspectos pedagógicos, a través de la enseñanza del docente y la construcción de un conocimiento o concepto en general que podemos asociarlo en la realidad que atravesamos día tras día, teniendo en cuenta objetivos y valores y en la satisfacción de conseguirlo, alcanzando los logros y superando obstáculos, obteniendo buenas calificaciones, mediante la tranquilidad de que el alumno pueda tener al saber contestar todas las dudas que pueda tener, llevando a que pueda aprender todos los contenidos correctamente; ver qué la institución en la que se estudia se preocupa por el real aprendizaje de sus alumnos, una buena predisposición de parte de profesores es esencial y finalmente, un alumno siente satisfacción cuando sus opiniones o propuestas son valoradas. En relación a la calidad del servicio, se coordinan y administran mecanismos de comunicación múltiples que permitan enriquecer los recursos del aprendizaje, a la vez que se formalizan las vías de comunicación bidireccionales y frecuentes relaciones de mediación, dinámicas e innovadoras, a los fines de elevar la calidad del mismo. Por otra parte, la calidad de dicho servicio, se eleva a la vez con mayor cantidad de clases virtuales; a través de la pedagogía y la didáctica con el alumno, mediante la búsqueda de materiales funcionales para la enseñanza y aprendizaje, en ellos motivarlos y poder alcanzar el objetivo que es aprender; usando herramientas informáticas de manera cotidiana; mejorando la comunicación y foros; mediante tele clases en las que el profesor realice las explicaciones y en las materias que requieren mayor práctica.

\section{Ante lo expuesto, las hipótesis planteadas, son aceptadas, ya que:}

- Existe una relación entre la comunicación interpersonal y el desarrollo del proceso de socialización entre alumnos, docentes, egresados, en la Universidad Privada Argentina, puesto que desde la organización escolar se incorporaron modelos comunicaciones adecuados a los avances tecnológicos actuales, mediante diseños de plataformas multimedia, que ofrecen calidad tanto en la estructura y diseño de los contenidos curriculares que se imparten y optimizan la planificación institucional. 
- Las estrategias innovadoras que se implementan a los fines de mejorar servicio educativo a distancia en la Universidad Privada de Argentina son aquellas tendientes a garantizar la participación de los actores de la comunidad educativa, la incorporación de herramientas tecnológicas para llevar adelante el proceso de enseñanza y aprendizaje, módulos de contenidos curriculares y el uso de estrategias metodológicas que favorezcan el aprendizaje autónomo, adecuación de las estrategias a la calidad del servicio brindado, haciendo de la plataforma virtual, funcional a los requerimientos de los usuarios.

Por lo tanto, se concluye que el proceso comunicativo en la educación ha cambiado, a través de nuevos medios comunicativos que generan a su vez, diferentes interacciones entre los docentes y los alumnos y el aula se transformada por la incorporación de elementos tecnológicos y la calidad de la misma, se debe al uso de los profesores de medios tecnológicos; se señala además que es fundamental el medio ambiente donde se lleva adelante la comunicación educativa, como así también se señala como fundamental dar o generar gran importancia al rol comunicativo en el proceso educativo, siendo una de las funciones básicas de los profesores la educativa. De tal manera, que la comunicación es de vital importancia porque permite la unión de los procesos tecnológicos y el desarrollo de los contenidos en la educación a distancia, puesto que la educación bajo esta modalidad posibilita la creación de ambientes donde el proceso de aprendizaje se centra en el alumno y se caracteriza por su interactividad, accesibilidad y eficiencia. $\mathrm{Y}$ es en este proceso en este proceso, donde surgen mecanismos de socialización como micro-procesos que dan lugar a la construcción social del alumno mediante el aprendizaje y la interiorización del contenido y la socialización se convierte en un proceso de aprendizaje que permite la adquisición de orientaciones adecuadas para desenvolverse adecuadamente en un rol.

\section{LISTA DE REFERENCIAS}

Abelson, H. (2007). TheCreation of OpenCourseWare at MIT, Journal of ScienceEducation and Technology, 17 (2), 164-174.

Acosta Peralta, M. (2009). La educación a distancia en la República Dominicana: Situación actual y desafíos futuros. Tesis doctoral sin publicar, Universidad Estatal a Distancia (UNED), San José, Costa Rica. 
Alfonzo Paradisi, A.J. (2011). Caracterización de algunas dimensiones de la interacción didáctica en la modalidad de educación a distancia. Caso: Universidad Nacional Abierta de Venezuela. Tesis doctoral sin publicar, Universidad Nacional de Educación a Distancia (UNED), Madrid, España.

Allen, I. E. y Seaman, J. (2003). Sizingtheopportunity: Thequality and extent of online education in theUnitedStates. Needham, MA, EE. UU.: TheSloanConsortium. Recuperado de: http://www.sloan-c.org/resources/sizing_opportunity.pdf.

Álvarez, J. M. (2014). Evaluar para conocer, examinar para excluir. Madrid: Morata.

Anderson, J.R. (2001). Aprendizaje y memoria: Un enfoque integral. México: McGrawHill.

Anderson, T., y Dron, J. (2011). Threegenerations of distanceeducationpedagogy. International Review of Research in Open and DistanceLearning, Vol.12, No.3.

Anderson, T. y Three, D. J. (2011). Generations of DistanceEducationPedagogy. International Review of Research in Open and DistanceLearning. Recuperado de http://www.irrodl.org/index.php/irrodl/article/view/890/1826;12.

Aparici, R. y Osuna-Acedo, S. (2013). La Cultura de la Participación. Revista Mediterránea de Comunicación, 4 (2), 137-148. Recuperado de: http://dx.doi.org/10.14198/MEDCOM2013.4.2.07.

Arboleda, N. (2002). ABC de la educación virtual y a distancia. Educación virtual. Bogotá: Interponed.

Assmann, H. (2002). Placer y ternura en educación. Madrid: Narcea.

Attwell, G. (2008). The Personal LearningEnvironments -thefuture of eLearning? En eLearning $\quad$ Papers. 2 (1). Recuperado de http://www.elearningeuropa.info/files/media/ media11561.pdf.

Barabási, A.L. (2013). Network science. PhilosophicalTransactions of The Royal Society, No.371, p.1-3.

Barbera, E. y Badia, A. (2004). Educar con Aulas Virtuales. Madrid: Antonio Machado Libros S.A.

Bautista, G.; Borges, F. y Fores, A. (2008). Didáctica universitaria en entornos virtuales de enseñanza y aprendizaje. Madrid: Narcea.

Berge, Z. L. (1995). The role of the online instructor/facilitator. EducationalTechnology, Vol.35, No.1, p.22-30. 
Bernal Álvarez, Yo. (1994). Importancia del estudio independiente en la educación abierta. Antología Módulo Propedéutico de Nivelación a Licenciatura en Trabajo Social, México: Universidad de Guadalajara. Guadalajara, Jalisco.

Bohm, D. (2013). On dialogue. New York: Routledge.

Burbules, N. (1999). El diálogo en la enseñanza. Buenos Aires: Amorrortu Editores.

Cabana, S.; Cortés, F.; Vega, D. y Cortés, R. (2016). Análisis de la fidelización del estudiante de Ingeniería con su centro de Educación Superior: Desafíos de Gestión Educacional. Formación Universitaria, vol. 9, nº 6, pp. 93 - 104, 2016.

Cárdenas Chávez, J. (1992). En búsqueda de un modelo de asesoría en Anguiano Molina, Ana María (2006). Antología Módulo Propedéutico de Nivelación a Licenciatura en Trabajo Social. México: Universidad de Guadalajara. Guadalajara, Jalisco.

Castells, M. (2005). Thenetworksociety: Fromknowledgetopolicy. En M. Castells y G. Cardoso (Eds.), Thenetworksociety: Fromknowledgetopolicy (pp.3-21). Washington, DC: Johns Hopkins Center forTransatlanticRelations.

CofánYong, E. (2014). Documento Maestro. Maestría en Gestión de la Educación Virtual. Colombia: Universidad EAN.

Coll, C. y Monereo, C. (2008). Psicología de la educación virtual. Conferencia mundial sobre la Educación Superior en el siglo XXI: visión y acción. Madrid: Morata.

Collis, B. (1995). FlexibleLearning in Digital WorldExpectation. Londres: Kogan Page.

Collis, B. y Moneen, J. (2001). Flexible Learning in a digital world. Londres: Kogan Page.

Connick, G. P. (1999). Thedistancelearner's guide. UpperSaddleRiver. NJ, EE. UU.: Prentice Hall.

Coutinho, V. (2017). Aprende todo sobre el marketing educativo y cómo atraer más alumnos a tu institución educativa. Recuperado de https://rockcontent.com/es/blog/marketing-educativo/.

Chatti, M. A. (2009). Mashup Personal LearningEnvironment. Recuperado de http://www.google.com/reader/shared/06179808011277023861.

De la Fuente Mella, H.; Marzo Navarro, M. y Reyes Riquelme, M. J. (2010). Análisis de la satisfacción universitaria en la Facultad de Ingeniería de la Universidad de Talca,» Revista chilena de ingeniería, vol. 18, n³ 3, pp. 350 - 363, 2010. 
Discenza, R.; Howard, C. y Schenk, K. (2001). Thedesign and management of effectivedistancelearningprograms. Nueva York: Idea Group Publishing.

Downes, S. (2010). Places togo: Connectivism\&connectiveknowledge. Innovate Recuperado de http://www.innovateonline.info/pdf/vol5_issue1/Places_to_Go_Connectivism_\&_Connective_Knowledge.pdf.

Driscoll, C.T. (2000). Constructivism: Theory, perspectives, and practice. New York: TeachersCollegePress, Columbia University.

EADBOX. (2018). Conoce las estrategias de marketing para cursos online y aumenta tus ventas. Recuperado de https://es.eadbox.com/estrategias-de-marketing-paracursos/.

Easton, S.S. (2003). Clarifyingtheinstructor's role in online distancelearning. Communication.

Elder, L. y Paul, R. (2010). The Role of SocraticQuestioning in Thinking, Teaching, and Learning. TheClearingHouse: A Journal of EducationalStrategies, Issues and Ideas, 71(5), 297-301. Recuperado de https://doi.org/10.1080/00098659809602729.

Encinas Orozco, F. C. y Cavazos Arroyo, J. (2017). Students' loyalty in highereducation: the roles of affectivecommitment, serviceco-creation and engagement. Cuadernos de Administración, vol. 33, nº 57, pp. 96 - 110, 2017.

Fainhloc, B. (1999). La intercatividad en la educación a distancia. Buenos Aires: Paidós.

Fernández-Abascal, E.; García Rodríguez, B.; Jiménez Sánchez, M. D. y Domínguez Sánchez, F. J. (2011). Psicología de la emoción. Madrid: Ramón Areces.

Flecha, R. (1997). Compartiendo Palabras: el aprendizaje de las personas adultas a través del diálogo. Barcelona: Paidós.

Freire, P. (1970). Pedagogía del Oprimido. Madrid: Siglo XXI.

Freire, P. y Faundez, A. (2013) Pedagogía de la pregunta. Buenos Aires: Siglo Veintiuno.

García, P. F. (2005). Estado actual de los sistemas e-learning. Teoría de la Educación: Educación y Cultura en la Sociedad de la Información, $\mathrm{N}^{\circ} 6$.

García Aretio, L. y Ruiz Corbella, M. (2010). La eficacia en educación a distancia: ¿Un problema resuelto? Teoría de la Educación (Universidad de Salamanca), Vol.22, No.1, p.141-162.

García Aretio, L. (2013). Objetivos y funciones de la educación a distancia. Madrid: UNED. 
García Llamas, J. L. (1986), Un modelo de análisis para la evaluación del rendimiento académico en la enseñanza a distancia, Madrid. OEI.

Garrison, D.R., Anderson, T., y Archer, W. (2000). Criticalinquiry in a textbasedenvironment: Computerconferencing in highereducation. The Internet and HigherEducation, Vol.2, No.2-3, p.87-105.

Garrison, D. R. (2007). Online community of inquiryreview: Social, cognitive, and teachingpresenceissues. Journal of AsynchronousLearning Networks, Vol.11, No.1, p. 61-72.

Gento Palacios, S., y Vivas García, M. (2003). EL SEUE: Un Instrumento para Conocer la Satisfacción de los Estudiantes Universitarios con su Educación. Acción Pedagógica, 12(2), 16-27.

Gómez, J. (2004). Las TIC en educación. Madrid: IES Gabriel García Márquez.

Greenhow, C.; Robelia, B. y Hughes, J. (2009). Learning, teaching, and scholarship in a digital age: Web 2.0 and classroomresearch: Whatpathshouldwetakenow?.EducationalResearcher. Recuperado de http://edr.sagepub.com/content/38/4/246.full.

Grow, G. (1991). TeachingLearnersto Be SelfDirect. AdultEducationQuartely, vol. XLI, $\mathrm{N}^{\circ} 3$, pp. 125-149. Tallahassee, Florida.

Gunawardena, C.N., y McIsaac, M.S. (2004). Distanceeducation. En: D.H. Jonassen (Ed.), Handbook of ResearchonEducationalCommunications and Technology (Mahwah, NJ: Lawrence Erlbaum.

Hittelman, M. (1998). Whydistanceeducationisnot inevitable forthecolleges. En K. Martinez (Ed.). FACCCTS: TheJournal of theFacultyAssociation of California CommunityColleges, 1997-1998, 4(1-4), 86-87.

Holmberg, B. (2003). Distanceeducation in essence: Anoverview of theory and practice in theearlytwenty-firstcentury. Oldenburg: Carl von OssietzkyUniversitätOldenburg (BIS)- Verlag.

Howell, S. L., Williams, P. B. y Lindsay, N. K. (2003). Thirtytwotrendsaffectingdistanceeducation: Aninformedfoundationforstrategicplanning. Online Journal of DistanceLearningAdministration, 6(3). Recuperado de: http://www.westga.edu/ distance/ojdla/fall63/howell63.html.

Kofman, F. (2003). Metamanagement. Buenos Aires: Ediciones Granica. 
Laurillard, D. (2002). RethinkingUniversityTeaching: A Conversational Framework fortheEffective Use of Learning Technologies, Segunda edición. London: RoutledgeFalmer.

Levine, A. y Sun, J. C. (2002). Barriers to distance education [Versiónelectrónica]. En Distributed Education: Challenges, Choices, and a New Environment: Vol. 6. Washington, D.C, EE. UU: American Council on Education / EDUCASE. Recuperado de: http://www.acenet.edu/bookstore/ pdf/distributedlearning/distributed-learning-06.pdf.

Lévy, P. (2004). Inteligencia colectiva: por una antropología del ciberespacio. Recuperado de: https://goo.gl/qe8MWw.

Lockwood, F. (1998). TheDesign and Production of Self-Instructional Material. Londres: Kogan Page.

López Rayón Parra, A. E.; Ledesma Saucedo, R.; Escalera Escajeda, S.; (2009). Ambientes virtuales de aprendizaje. Recuperado de http://investigacion.ilce.edu.mx/panel_control/doc/Rayon_Parra.pdf.

Lozano, J. (2004). El Triángulo del E-learning. Noticias.com.

Lubensky, R. (2006). Thepresent and future of Personal LearningEnvironments (PLE).

Recuperado de http://members.optusnet.com.au/rlubensky/2006/12/present-andfuture-ofpersonal-learning.html.

Manes, J. M. (2000). Ética y Marketing de la Institución Educativa: una conciliación. Recuperado de http://contexto-educativo.com.ar/2000/3/nota-4.htm.

Manuale, M. (2013). El oficio del estudiante universitario y el problema del ingreso. Miradas y aportes desde el Gabinete Pedagógico de la Facultad de Bioquímica y Ciencias Biológicas. Revista Aula Universitaria 15. Año 2013. (pp. 43-57). Recuperado de: bibliotecavirtual.unl.edu.ar/.

Marín Ibáñez, R. (1984). El sistema pedagógico de la UNED y su rendimiento, en Evaluación del rendimiento de la enseñanza superior a distancia. Madrid: UNED. Marqués, P. (2000a). Impacto de las TIC en educación. Funciones y limitaciones. Material de enseñanza. Barcelona: Universidad Autónoma de Barcelona. Facultad de Educación, Departamento de Pedagogía Aplicada. 
Marqués, P. (2000b). Las TIC y sus aportaciones a la sociedad. Material de enseñanza.

Barcelona: Universidad Autónoma de Barcelona, Facultad de Educación, Departamento de Pedagogía Aplicada.

Marta-Lazo, C. y Gabelas, J. A. (2016). Comunicación Digital. Un modelo basado en el factor Relacional. Barcelona: Editorial UOC.

Marta-Lazo, C.; Osuna-Acedo, S. y Frau-Meigs, D. (2018). Collaborativelifelonglearning and professional transfer. Case study: ECO European Project. InteractiveLearningEnvironments, 26, 1-14. Recuperado de https://doi.org/10.1080/10494820.2018.1451346.

Mestres, L. (2007). Los medios de comunicación y la educación. Recuperado de https://www.educaweb.com/noticia/2007/02/12/medios-comunicacion-educacion$2206 /$.

Mestres, L. (2010). Cómo iniciar una estrategia de marketing educativo. Recuperado de https://www.educaweb.com/noticia/2010/11/02/como-iniciar-estrategiamarketing-educativo-4453/.

Mestre, L. (2014). Estrategias de fidelización del alumnado. Recuperado de https://www.educaweb.com/noticia/2014/03/11/estrategias-fidelizacionalumnado-8090/.

Moore, M.G. (1993). Theory of transactionaldistance. En: D. Keegan (Ed.), Theoreticalprinciples of distanceeducation (pp.22-38). Londres, Inglaterra: Routledge.

Morán, L. y Myringer, B. (1999). Flexible learning and universitychange. En Harry,K. (ed.). HigherEducationThrough Open and DistanceLearning. Londres: Routledge, $57-72$.

Moreira, A. M. (2012). Alfabetización digital y competencias informacionales. Fundación Telefónica. Recuperado de: ddv.stic.ull.es/.

Nicol, D. J. y Macfarlane-Dick, D. (2006). Formativeassessment and selfregulatedlearning: A model and sevenprinciples of goodfeedbackpractice. Studies in HigherEducation, 31(2): 199-218. Recuperado de https://doi.org/10.1080/03075070600572090.

Odin, J. K. (2002). Teaching and learning activities in the online classroom: A constructivist perspective. Denver, CO, EE. UU. 
Oliver, R. (2003). Exploringbenchmarks and standardsforassuringquality online teaching and learning in highereducation. Proceedings of 16th Open and DistanceLearningAssociation of Australia BiennialForum, Canberra, Australia, Octubre, p.79-90.

Open Licensing (OCL). for Open Educational Resources. Recuperado de http://www.oecd.org/edu/oer.

Pacheco Cortés, A. (2002). Cursos en línea en Revista Apertura No. 2 Innovación para el aprendizaje. México: U de G. Guadalajara, Jalisco.

Pagano, C.M. (2007). Los tutores en la educación a distancia: Unaporteteórico. Revista de Universidad y SociedaddelConocimiento, Vol.4, No.2.

Paul, R. y Elder, L. (2003). La mini-guía para el pensamientocrítico, conceptos y herramientas. California: Fundación para el pensamientocrítico.

Perazo, M. I. (1981. Educación a Distancia. Buenos Aires: Asociación Argentina de Educación a Distancia.

Pérez Juste, R. (2003). La calidad de la educación. Hacia una educación de calidad. Gestión, instrumentos y evaluación. Madrid: Narcea.

Perrenoud, P. (2006). El oficio de alumno y el sentido del trabajo escolar. Madrid: Editorial Popular.

Piccoli, G.; Ahmad, R. e Blake, I. (2001). Web-Based Virtual LearningEnviroments: A Research Framework and a PreliminaryAssessment of Effectiveness in Basic IT Skills Training. MIS Quarterly, vol. XXV, N 4, pp. 401-426.

Pretella, C. (2008). Gestión de la relación de las universidades con docentes, estudiantes y egresados. Revista Iberoamericana de Educación, vol. 5, nº 47, Noviembre 2008.

Prieto Castillo, D. y Van de Pol, P. (2006). e-Learningcomunicación y educación El diálogo continúa en el ciberespacio. San José, Costa Rica: Radio Nederland Training Centre.

Programa de las Naciones Unidas para el Desarrollo (PNUD). (2002). Las tecnologías de la información y la comunicación al servicio del desarrollo. Informe sobre Desarrollo Humano. Venezuela.

Rama, C. (2013). El constructo calidad. Definición y dimensiones. Conferencia dictada en el II Taller Centroamericano de Armonización de Componentes Conceptuales y Técnicos: Estándares y criterios calidad, para las agencias de acreditación 
regionales de la educación superior. Panamá: Consejo Centroamericano de Acreditación de la Educación Superior (CCA).

Rojas, A. R.; Corral, R.; Alfonso, I. y Ojalvo, V. (2000). La tecnología educativa. El uso de las NTIC en la educación. En: Universidad de La Habana. Tendencias pedagógicas en la realidad educativa actual. Tarija: Editorial Universitaria. Universidad Juan Misael Saracho.

Rosemberg, M. (2001). E-learningStrategiesforDeliveringKnowledge in the Digital Age. Nueva York: McGraw-Hill.

Roura-Redondo, M. (2017). Aprendizaje dialógico en escenarios virtuales: Percepción de la comunidad universitaria sobre el diálogo online. Revista Mediterránea de Comunicación/MediterraneanJournal of Communication, 8(2), 287-300. Recuperado de https://www.doi.org/10.14198/MEDCOM.

Roura-Redondo, M.; Camarero, L. y Osuna-Acedo, S. (2018). La evaluación para aprender (EpA) y el empoderamiento de los estudiantes. En J. Rodríguez Terceño (coord.), Investigando en Comunicación e Investigando en Docencia (pp. 411-423) Madrid: Tecnos.

Rumble, G. (1989). Ondefiningdistanceeducation. The American Journal of DistanceEducation, Vol.3, No.2, p.8-21.

Salgado García, E. (2015). La enseñanza y el aprendizaje en modalidad virtual desde la experiencia de estudiantes y profesores de posgrado. Tesis de Doctorado. San José, Costa Rica: Universidad Católica de Costa Rica.

Sacristán Romero, F. (2006). Marco actual de la educación a distancia. Revista Espacio Académico 2006; 4(16). Recuperado de: http://www.rpbahia.com.br/revista/marco_actual_de_la_educacion_a_distancia.pdf.

Salinas, J. (1997). Nuevos ambientes de aprendizaje para una sociedad de la información. Revista Pensamiento Educativo. 1997; 20:81-104.

Sampieri, R. (2014). Metodología de la investigación. México: McGraw Hill.

Segura, S. (2004). Modelo comunicativo de la educación a distancia apoyada en las TIC en la Corporación Universitaria Autónoma de Occidente, CUAO, Cali- Colombia", Edutec. Revista Electrónica de Tecnología Educativa, núm. 17. Recuperado de http://edutec.rediris.es/Revelec2/revelec17/segura_16a.pdf. 
Shin, N. (2002). Beyondinteraction: Therelationalconstruct of 'transactionalpresence'. Open Learning, Vol.17, No.2. doi: 10.1080/02680510220146887.

Siemens, G. (2005). Connectivism: A learningtheoryforthe digital age. International Journal of InstructionalTechnology and DistanceLearning, Vol.2, No.1, enero. Recuperado de http://www.itdl.org/Journal/Jan_05/article01.htm.

Spiro, B.F. (2011). Thetechnology of teaching. New York: Appleton-Century-Crofts.

Suárez, C. (2004, junio). La zona de desarrollo próximo, categoría pedagógica para el análisis de la interacción en contextos de virtualidad, Píxel-Bit, Revista de Medios y Comunicación, núm. 24. Recuperado de http://www.sav.us.es/pixelbit/articulos/n24/n24art/art2401.htm.

Tintaya, E. (2003). Desafíos y fundamentos de educación virtual. Material de enseñanza. Bolivia: Universidad Mayor San Andrés, Ciencias de la Educación.

Touriñán, J. M. (1996). Educación y sociedad de \& información: Cuestiones estratégicas para el desarrollo de propuestas pedagógicas. Santiago de Compostela: ICEUniversidade de Santiago de Compostela.

Touriñán, J. M. (2001). Sociedad de la información y cooperación al desarrollo: una posición de valor en los sistemas educativos. Santiago de Compostela: IcEUniverdidade de Santiago de Compostela, 2000, pp. 183-214.

UNCU Virtual (2018). Estrategias. Recuperado de http://uncuvirtual.uncuyo.edu.ar/4equipos-de-trabajo.

Van den Brande, L. (1993). Flexible and DistanceLearning. Chicherter (UK): John Wiley\&Sons.

Vásquez, R.; Bongianino, C. y Sosisky, L. (2006). La tecnología educativa y la educación a distancia. Educación a distancia de grado y pregrado. XVII Jornadas Universitarias de Contabilidad. Argentina: Universidad Nacional de Entre Ríos.

Vizcaíno, A. (2007). La asesoría académica y el rol docente en la educación a distancia. Recuperado de https://educrea.cl/la-asesoria-academica-y-el-rol-docente-en-laeducacion-a-distancia/.

UNED (2010). Educación a Distancia y Formación Virtual. Recuperado de http://portal.uned.es/portal/page?_pageid=93,56233773\&_dad=portal\&_schema= PORTAL\&idAsignatura=23302263. 
Wagner, J. G. (2001). Assessing online learning. National Business EducationAssociation: Keying In, 11(4).

Webster, J. y Hackley, P. (1997). Teachingeffectiveness in technologymediateddistancelearning. TheAcademy of Management Journal, Vol XL No 6 , pp. 1282-1309.

Weisinger, H. (1998) La inteligencia emocional en el trabajo. Buenos Aires, Argentina: Javier Vergara Editores. 